Published in final edited form as:

Curr Genet. 2016 May ; 62(2): 343-346. doi:10.1007/s00294-015-0553-3.

\title{
The exocyst in Candida albicans polarized secretion and filamentation
}

\author{
Alba A. Chavez-Dozal ${ }^{1,2}$, Stella M. Bernardo ${ }^{1,2}$, and Samuel A. Lee Le, $^{1,2}$ \\ ${ }^{1}$ Section of Infectious Diseases, New Mexico VA Healthcare System, 1501 San Pedro SE, Mail \\ Code 111-J, Albuquerque, NM 87108, USA \\ 2 Division of Infectious Diseases, University of New Mexico Health Science Center, Albuquerque, \\ NM 87131, USA
}

\begin{abstract}
The exocyst is an octameric complex that orchestrates the docking and tethering of vesicles to the plasma membrane during exocytosis and is fundamental for key biological processes including growth and establishment of cell polarity. Although components of the exocyst are well conserved among fungi, the specific functions of each component of the exocyst complex unique to Candida albicans biology and pathogenesis are not fully understood. This commentary describes recent findings regarding the role of exocyst subunits Sec6 and Sec15 in C. albicans lamentation and virulence.
\end{abstract}

\section{Keywords}

Exocyst; Filamentation; Polarisome; SEC6; SEC15; Secretion; Spitzenkörper

\section{Commentary}

Candida albicans is an important opportunistic fungal pathogen, and is the fourth most common cause of bloodstream infections in hospitalized patients in the U.S. (Hidron et al. 2008; Morgan et al. 2005). Invasive candidiasis is responsible for substantial morbidity and mortality (Morgan et al. 2005). C. albicans is uniquely adapted to mammalian commensalism, yet has the ability to lament and cause virulence as an opportunistic pathogen (Dujon 2010). While C. albicans and the model yeast Saccharomyces cerevisiae share a large number of predicted genes overall, there are significant differences in their genomes, including differences in size, ploidy, codon translation, and gene family expansions (Dujon 2010). Notably, it has become evident that nonhomologous genes in these yeasts have convergent functions, and similar orthologs have divergent functions with a remarkable degree of transcriptional re-wiring evident (Whiteway et al. 1992). Even minor differences, such as in yeast secretion, which is highly conserved in eukaryotic cells, lead to distinctly different phenotypes (Delic et al. 2013). The exocyst complex, which has been extensively studied in S. cerevisiae, is an evolutionarily well-conserved octameric complex

\footnotetext{
`Communicated by M. Kupiec. Alba A. Chavez-Dozal albitach@nmsu.edu, Samuel A. Lee SamALee@salud.unm.edu.
} 
involved in the final stages of secretion. Exocyst subunits and related proteins involved in exocytosis are well conserved among lamentous fungi; however, there is increasing evidence of distinct functional roles of exocyst subunits in C. albicans that are related to virulence, unveiling the complex nature of this fungal pathogen.

Polarized secretion is the key cellular process by which secretory cargo is transported in vesicles and directed to specific sites in the plasma membrane to facilitate fundamental cellular functions such as cell growth, morphogenesis, and cytokinesis (Heider and Munson 2012). Processes such as the asymmetric formation of a yeast bud prior to mitosis and cytokinesis to produce a daughter cell, formation of hyphae in response to environmental stimuli, and delivery of basolateral and apical proteins in a mammalian intestinal epithelial cell are all dependent on polarized secretion (Heider and Munson 2012; TerBush et al. 1996). The final stages of secretion that lead to exocytosis, depend on fusion of late secretory vesicles to the plasma membrane which is regulated by the exocyst complex (TerBush et al. 1996). This octameric complex mediates tethering of late secretory vesicles to the plasma membrane, followed by membrane fusion enabled by assembly and disassembly of a SNARE complex (Novick et al. 1981). In S. cerevisiae, the exocyst is encoded by SEC3, SEC5, SEC6, SEC8, SEC10, SEC15, EXO70, and EXO84 (Hsu et al. 2004).

Proper localization of the exocyst is dependent on SEC3 in an actin independent manner (Luo et al. 2014). Exo70 also contributes to exocyst localization in a partially actindependent manner, and the association of Sec3 and Exo70 is thought to be responsible for proper localization of the remainder of the exocyst complex (Boyd et al. 2004). Recruitment of Sec3 and Exo70 is dependent on PI(4,5) P2 (phosphatidylinositol 4,5-bisphosphate) in the plasma membrane, and regulatory proteins Cdc42 and the Rho1 GTPase (Boyd et al. 2004). Vesicle fusion to the plasma membrane is then mediated by binding between specific pairs of cognate v-SNAREs and t-SNAREs on the vesicle and target membranes (Marash and Gerst 2001). Rab GTP-binding proteins are required to facilitate formation of v-SNARE/tSNARE complexes (Marash and Gerst 2001). The t-SNAREs Snc1/2 and the v-SNAREs Sso1/2 each contribute to one helix, whereas the v-SNARE Sec9 contributes to two helices to the SNARE complex (Marash and Gerst 2001; Sutton et al. 1998). Tethering of the vesicle to the exocyst occurs first and is required for subsequent SNARE assembly, which then permits the fusion of the vesicle and target membranes to permit final exocytosis (Sutton et al. 1998). The exocyst component Exo84p also interacts directly with Sro7, which binds to and activates the t-SNARE Sec9 (Hattendorf et al. 2007). Exocytosis is profoundly defective in S. cerevisiae sro7 temperature-sensitive mutants. Sro7 likely functions directly in late secretion by interacting with the activated (GTP-bound) form of Sec4, thereby providing a functional link between vesicle arrival and assembly of the SNARE complex (Hattendorf et al. 2007).

Previous studies have emphasized the importance of exocyst components in C. albicans cell biology. Mutants of the $\mathrm{C}$. albicans exocyst display distinct phenotypes, including defects in bio $\mathrm{lm}$ formation, lamentation and secretion (Fig. 1). A C. albicans sec3 null mutant exhibited increased cell size and was unable to maintain tip growth after assembly of the first septin ring (Li et al. 2007). Conditional mutants lacking EXO84 were inviable after $24 \mathrm{~h}$ 
under repressing conditions; prior to cell death, mutant cells were enlarged and contained multiple nuclei. When mutants were grown on lamentation-inducing media, pseudohyphal growth was predominant in this conditional mutant (Caballero-Lima and Sudbery 2014). We have recently studied the role of several $\mathrm{C}$. albicans exocyst-related SNARE complexes. We demonstrated that the t-SNARE proteins Sso2 and Sec9 contribute to lamentation and required for aspartyl proteinase and lipase secretion (Bernardo et al. 2014). Filamentation is a multifactorial process that involves the orchestrated supply of secretory vesicles for polarized growth and hyphal expansion; post-Golgi vesicles travel to the hyphae along actin cables that become nucleated at the sites of polarized growth, a process that is facilitated by the polarisome, Spitzenkörper and the exocyst complex prior to fusion with the plasma membrane. Interestingly, the C. albicans Spitzenkörper dissipates in the absence of Sso2 (Bernardo et al. 2014), emphasizing the importance of this t-SNARE protein in C. albicans lamentation.

We next studied the role of SEC6 and SEC15 in C. albicans secretion and lamentation. We constructed two tetracycline regulatable strains, tetR-SEC6 and tetR-SEC15. Strain tetRSEC6 grown under repressing conditions had readily apparent defects in cytokinesis and endocytosis, and accumulated both post-Golgi secretory vesicles and structures suggestive of late endosomes (Chavez-Dozal et al. 2015a, b). Strains tetR-SEC6 and tetR-SEC15 were both markedly defective in secretion of aspartyl proteases and lipases (Chavez-Dozal et al. 2015a, b). In the tetR-SEC6 strain there was aberrant localization of chitin at the cell septum, and increased resistance to zymolyase and chitinase activity, suggesting that C. albicans Sec6 plays an important role in mediating trafficking and delivery of cell wall components. The tetR-SEC6 mutant was also defective in macrophage killing, indicating a role of SEC6 in C. albicans virulence (Chavez-Dozal et al. 2015a, b). Lack of SEC6 expression resulted in substantially reduced lateral hyphal branching, which requires the establishment of a new axis of polarized secretion; surprisingly, lack of SEC15 resulted in a hyper-branched phenotype (Chavez-Dozal et al. 2015a, b). Taken together, these studies indicate that the late secretory proteins Sec6p and Sec15p are required for polarized secretion and hyphal morphogenesis in C. albicans.

Given this direct contrast in hyphal branching phenotypes, we further studied the localization of Mlc1-GFP (marking the Spitzenkörper), Spa2-GFP (marking the polarisome), and Exo70-GFP (marking the exocyst) in the tetR-SEC15 and tetR-SEC6 mutants during lamentation (Chavez-Dozal et al. 2015a, b). The current model for polarized secretion in S. cerevisiae indicates that exocyst subunits travel to the plasma membrane via secretory vesicles that are transported along with actin cables. In the model proposed by Jones and Sudbery, during C. albicans polarized secretion in hyphal formation, a stream of vesicles arrive at the tip of the hyphae and accumulate in the Spitzenkörper; vesicles are then recruited by the polarisome and transported to cell surface where they dock with the exocyst (Jones and Sudbery 2010). In C. albicans hyphal growth, Mlc1-GFP is visualized as a subapical spot just behind the advancing tip of the hyphae, whereas Spa2-GFP and Exo70GFP localize to a crescent at the hyphal tip. In the tetR-SEC6 mutant, Mlc1-GFP, Spa2-GFP and Exo70-GFP displayed wild-type localization, whereas all of these structures were mislocalized in the tetR-SEC15 mutant. Moreover, upon alleviation of SEC15 gene repression, localization of Mlc1-GFP, Spa2-GFP, and Exo70-GFP was recovered in a 
sequential manner. Specifically, Mlc1-GFP recovered wild-type localization at $5 \mathrm{~min}$, Spa2GFP recovered after $10 \mathrm{~min}$, and Exo70-GFP recovered after $15 \mathrm{~min}$ (Chavez-Dozal et al. 2015a, b), revealing unique cellular dynamics of these key secretory structures.

These studies of SEC6 and SEC15 have led to some interesting findings that remain unexplained. It is not clear why the tetR-SEC6 and tetR-SEC15 mutant have differing phenotypes in hyphal branching and Spitzenkörper, polarisome, and exocyst localization. These experiments, however, were not able to definitively exclude the trivial explanation that transcript and/or protein dynamics were responsible for these apparently contrasting hyphal phenotypes. Specifically, since the tetR-SEC15 mutant became inviable at a much earlier time point than the tetR-SEC6 mutant, these differences in lamentation may simply be due to differences in exocyst kinetics rather than specific differences in subunit function. It is also possible that the slow depletion of SEC6 RNA may permit time for a compensatory response in cell wall integrity to occur, whereas the loss of SEC15 RNA occurs much more rapidly and thus there could be insufficient time for a compensatory response to occur. Alternatively, there may be as of yet unidentified compensatory proteins that assist the tetRSEC6 mutant in maintaining proper lamentation. Of great interest is the kinetics of Spitzenkörper, polarisome, and exocyst localization in the setting of loss of SEC15 expression and subsequent recovery. Presumably, given the rapidity of localization recovery, the key proteins for these structures remain present and readily accessible near the hyphal tip; when SEC15 expression resumes, these proteins then re-assemble in a sequential, coordinated fashion. The mechanisms of interactions between components of Spitzenkörper, polarisome, and exocyst with Sec15 remain unknown, and are the subject of further investigation in our laboratory.

While the exocyst complex is evolutionarily conserved in eukaryotic cells, these studies provide an initial glimpse of potential pathogen-specific roles of the exocyst in the polymorphic fungus $\mathrm{C}$. albicans.

\section{Acknowledgments}

This work was supported by funding from the Department of Veterans' Affairs (Merit Award 5I01BX001130 to SAL), Biomedical Research Institute of New Mexico (SAL), NIH Grant T32AI007538 (SMB), NIH Grant K12GM088021 (ASERT/IRACDA to AAC-D) and NIH Grant 5 T32 AI007538-13 (AAC-D).

\section{References}

Bernardo SM, Rane HS, Chavez-Dozal A, Lee SA. Secretion and lamentation are mediated by Candida albicans t-SNAREs Sso2 p and Sec9p. FEMS Yeast Res. 2014 doi: 10.1111/1567-1364.12165.

Boyd C, Hughes T, Pypaert M, Novick P. Vesicles carry most exocyst subunits to exocytic sites marked by the remaining two subunits, Sec3p and Exo70p. J Cell Biol. 2004; 167:889-901. [PubMed: 15583031]

Caballero-Lima D, Sudbery PE. In Candida albicans, phosphorylation of Exo84 by Cdk1-Hgc1 is necessary for efficient hyphal extension. Mol Biol Cell. 2014; 25:1097-1110. [PubMed: 24501427]

Chavez-Dozal AA, Bernardo SM, Rane HS, Herrera G, Kulkarny V, Wagener J, Cunningham I, Brand AC, Gow NA, Lee SA. The Candida albicans exocyst subunit Sec6 contributes to cell wall integrity and is a determinant of hyphal branching. Eukaryot Cell. 2015a; 14:684-697. [PubMed: 26002719] 
Chavez-Dozal A, Bernardo SM, Rane H, Lee SA. A functional analysis of the exocyst subunit Sec15 in Candida albicans. Eukaryot Cell. 2015

Delic M, Valli M, Graf AB, Pfeffer M, Mattanovich D, Gasser B. The secretory pathway: exploring yeast diversity. FEMS Microbiol Rev. 2013; 37:872-914. [PubMed: 23480475]

Dujon B. Yeast evolutionary genomics. Nat Rev Gen. 2010; 11:512-524.

Hattendorf DA, Andreeva A, Gangar A, Brennwald PJ, Weis WI. Structure of the yeast polarity protein Sro7 reveals a SNARE regulatory mechanism. Nature. 2007; 446:567-571. [PubMed: 17392788]

Heider MR, Munson M. Exorcising the exocyst complex. Traffic. 2012; 13:898-907. [PubMed: 22420621]

Hidron AI, Edwards JR, Patel J, Horan TC, Sievert DM, Pollock DA, Fridkin SK. NHSN annual update: antimicrobial-resistant pathogens associated with healthcare-associated infections: annual summary of data reported to the national healthcare safety network at the centers for disease control and prevention, 2006-2007. Infect Control Hosp Epidemiol. 2008; 29:996-1011. [PubMed: 18947320]

Hsu SC, TerBush D, Abraham M, Guo W. The exocyst complex in polarized exocytosis. Int Rev Cytol. 2004; 233:243-265. [PubMed: 15037366]

Jones LA, Sudbery PE. Spitzenkorper, exocyst, and polarisome components in Candida albicans hyphae show different patterns of localization and have distinct dynamic properties. Eukaryot Cell. 2010; 10:1455-1465. [PubMed: 20693302]

Li CR, Lee RT, Wang YM, Zheng XD, Wang Y. Candida albicans hyphal morphogenesis occurs in Sec3p-independent and Sec3p-dependent phases separated by septin ring formation. J Cell Sci. 2007; 120:1898-1907. [PubMed: 17504812]

Luo G, Zhang J, Guo W. The role of Sec3p in secretory vesicle targeting and exocyst complex assembly. Mol Biol Cell. 2014; 25:3813-3822. [PubMed: 25232005]

Marash M, Gerst JE. t-SNARE dephosphorylation promotes SNARE assembly and exocytosis in yeast. EMBO J. 2001; 20:411-421. [PubMed: 11157748]

Morgan J, Meltzer MI, Plikaytis BD, Sofair AN, Huie-White S, Wilcox S, Harrison LH, Seaberg EC, Hajjeh RA, Teutsch SM. Excess mortality, hospital stay, and cost due to candidemia: a casecontrol study using data from population-based candidemia surveillance. Infect Control Hosp Epidemiol. 2005; 26:540-547. [PubMed: 16018429]

Novick P, Ferro S, Schekman R. Order of events in the yeast secretory pathway. Cell. 1981; 25:461469. [PubMed: 7026045]

Sutton RB, Fasshauer D, Jahn R, Brunger AT. Crystal structure of a SNARE complex involved in synaptic exocystosis at 2.4 A resolution. Nature. 1998; 395:347-353. [PubMed: 9759724]

TerBush DR, Maurice T, Roth D, Novick P. The exocyst is a multiprotein complex required for exocytosis in Saccharomyces cerevisiae. EMBO J. 1996; 15:6483-6494. [PubMed: 8978675]

Whiteway M, Dignard D, Thomas DY. Dominant negative selection of heterologous genes: isolation of Candida albicans genes that interfere with Saccharomyces cerevisiae mating factor-induced cell cycle arrest. Proc Natl Acad Sci. 1992; 89:9410-9414. [PubMed: 1409649] 


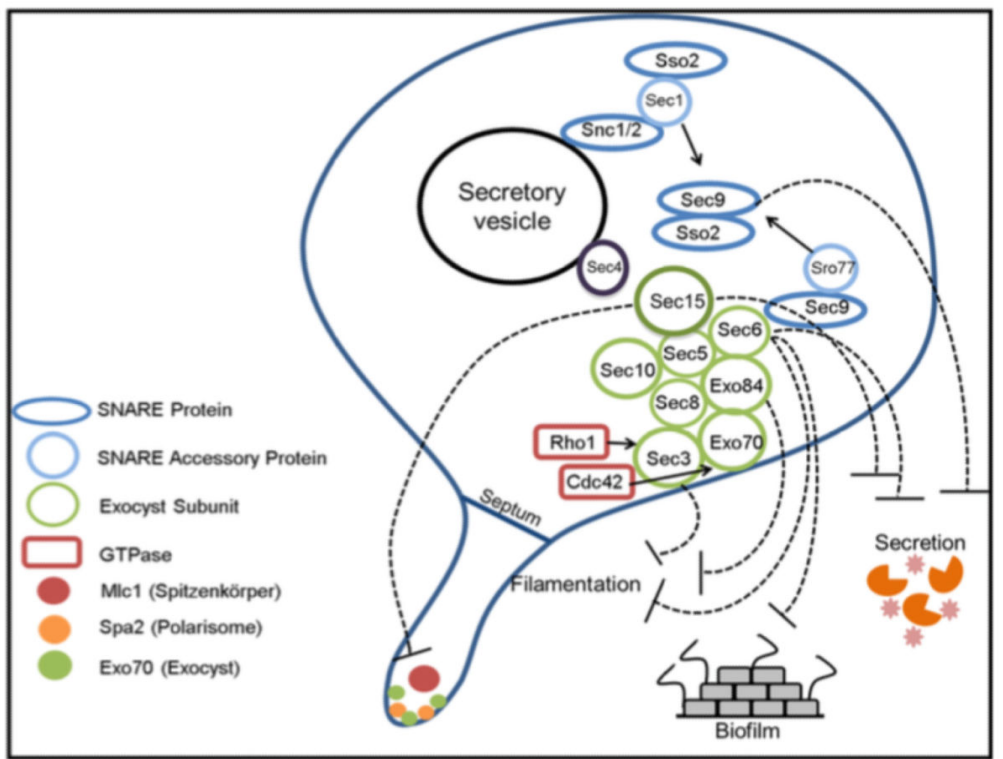

Fig. 1. Role of exocyst components in C. albicans secretion, lamentation, and biofilm formation Loss of Sec6, Sec15 and the SNARE proteins Sso2 and Sec9 interferes with secretion of proteases and lipases (indicated by dotted lines). Loss of Sec6 interferes with lamentation. Mutants lacking Sec6, Sec15, Exo84, Sso2, or Sec9 have impaired lamentation. Loss of Sec3 inhibits germ tube formation after septum formation. Loss of Sec15 interferes with Mlc1-GFP, Spa2-GFP, and Exo70-GFP localization. 